OPEN ACCESS

Edited by:

Alexander J. Shackman,

University of Maryland, USA

Reviewed by:

Tim Salomons,

University of Reading, UK

Sara E. Berger,

Northwestern University, USA

Sandra Kamping,

University Medical Centre Goettingen and Georg-August University,

Germany

*Correspondence:

Michael L. Meier

Balgrist University Hospital,

Forchstrasse 340, 8008 Zurich,

Switzerland

michael.meier@uzh.ch

Received: 25 March 2015 Accepted: 10 July 2015

Published: 24 July 2015

Citation:

Meier ML, Stämpfli P, Vrana A,

Humphreys BK, Seifritz $E$ and Hotz-Boendermaker S (2015)

Fear avoidance beliefs in back pain-free subjects are reflected by

amygdala-cingulate responses.

Front. Hum. Neurosci. 9:424. doi: 10.3389/fnhum.2015.00424

\section{Fear avoidance beliefs in back pain-free subjects are reflected by amygdala-cingulate responses}

\author{
Michael L. Meier ${ }^{1,2 *}$, Phillipp Stämpfli3,4, Andrea Vrana' ${ }^{1}$ Barry K. Humphreys ${ }^{1}$, \\ Erich Seifritz ${ }^{3}$ and Sabina Hotz-Boendermaker ${ }^{3}$ \\ ${ }^{1}$ Balgrist University Hospital, Zurich, Switzerland, ${ }^{2}$ Center of Dental Medicine, University of Zurich, Zurich, Switzerland, \\ ${ }^{3}$ Department of Psychiatry, Psychotherapy and Psychosomatics, Hospital of Psychiatry, University of Zurich, Zurich, \\ Switzerland, ${ }^{4}$ MR-Center of the Psychiatric Hospital and Department of Child and Adolescent Psychiatry, University of \\ Zurich, Zurich, Switzerland
}

In most individuals suffering from chronic low back pain, psychosocial factors, specifically fear avoidance beliefs (FABs), play central roles in the absence of identifiable organic pathology. On a neurobiological level, encouraging research has shown brain system correlates of somatic and psychological factors during the transition from (sub) acute to chronic low back pain. The characterization of brain imaging signatures in pain-free individuals before any injury will be of high importance regarding the identification of relevant networks for low back pain (LBP) vulnerability. Fear-avoidance beliefs serve as strong predictors of disability and chronification in LBP and current research indicates that back pain related FABs already exist in the general and pain-free population. Therefore, we aimed at investigating possible differential neural functioning between high- and low fear-avoidant individuals in the general population using functional magnetic resonance imaging. Results revealed that pain-free individuals without a history of chronic pain episodes could be differentiated in amygdala activity and connectivity to the pregenual anterior cingulate cortex by their level of back pain related FABs. These results shed new light on brain networks underlying psychological factors that may become relevant for enhanced disability in a future LBP episode.

Keywords: fear avoidance, low back pain, amygdala, pgACC, vulnerability, fMRI, PPI analysis, chronic low back pain

\section{Introduction}

Acute low back pain (LBP) has a favorable prognosis; most patients recover within 6 weeks (Koes et al., 2001). However, a small minority of patients develop disabling persistent and/or recurrent LBP that accounts for a considerable burden in terms of pain and suffering, loss of productivity and substantial health care expenditures (Bronfort et al., 2004; Rapoport et al., 2004; Peterson et al., 2012). In the development of chronic disability and in the absence of identifiable organic pathology, psychosocial variables, specifically fear avoidance beliefs (FABs), have been recognized as significant prognostic factors (Vlaeyen and Linton, 2000; Buchbinder et al., 2001; Buer and Linton, 2002; Leeuw et al., 2007b; Chou and Shekelle, 2010; Wertli et al., 2013). Such fears are represented by a subjectively misinterpreted importance of back pain and an associated vulnerability of the spine and can lead to avoidance behavior due to fear that certain behaviors will worsen the pain. Excessive FABs result in heightened disability 
and are an obstacle for recovery from acute, subacute, and chronic LBP (Rainville et al., 2011).

From a neurobiological perspective, the characterization of brain imaging signatures in pain-free individuals before any injury will be crucial if we are to identify the relevant networks for (back) pain chronicity and associated disability (Dunn et al., 2013; Denk et al., 2014). Recent research has narrowed the characterization of brain signatures involved in each stage of the disease and in the transition from (sub-) acute to chronic LBP (Baliki et al., 2012). Hashmi et al. (2013) have convincingly shown that during the transition from acute to chronic back pain, brain activity related to the perception of back pain shifted from regions linked to nociception to brain networks associated with emotion. In addition to these functional abnormalities, structural abnormalities, such as white matter abnormalities, seem to play an important role during the chronification process (Mansour et al., 2013). Patients have shown decreases in gray matter density in areas associated with pain processing (Ivo et al., 2013; Lloyd et al., 2014). At the stage of chronic LBP, these brain changes can show discriminative power leading to the ability to distinguish between individuals with and without chronic LBP on a neural level (Callan et al., 2014; Ung et al., 2014).

However, the mechanisms underlying these brain changes are still unclear. Although FABs have found broad empirical support as a predictor of chronic LBP and associated disability, neural substrates of the fear component could not yet be demonstrated when comparing chronic LBP patients and painfree controls (Barke et al., 2012). Regarding pain-free controls, there is evidence that back pain related FABs already exist in the healthy population (Buer and Linton, 2002). Therefore, we aimed at investigating possible differential neural functioning between high- and low fear-avoidant individuals in the general population using functional magnetic resonance imaging (fMRI). Risk assessment was carried out by means of the adapted version of the Tampa Scale for Kinesiophobia (TSK for the general population, TSK-G) questionnaire. The TSK-G measures FABs in the general population by specifically focusing on movementrelated fear of pain (Houben et al., 2005). During the fMRI measurements, subjects observed randomly presented video clips of daily activities that have been described as being perceived as either harmful or harmless for the back (Leeuw et al., 2007a).

On the neural level, we primarily focused on amygdala activity and connectivity because this brain area represents a key region in the evaluation and representation of fear intensity and pain and in deciphering threats in visual scenes (Kryklywy et al., 2013). Furthermore, amygdala activity and its functional connectivity to the prefrontal cortex (PFC) have been shown to be meaningfully different between individuals with different emotional modulation strategies and are strongly related to treatment response in chronic pain conditions (Bushnell et al., 2013; Silvers et al., 2014; Simons et al., 2014b). We hypothesized that there would be differential amygdala activity and task-related connectivity in individuals with high TSK-G scores compared to those with low TSK-G scores. Such a finding could reflect brain network properties underlying psychological factors that may become relevant for enhanced disability in a future LBP episode.

\section{Materials and Methods}

\section{Subjects and Questionnaires}

Following an online advertisement, 28 healthy subjects (15 females, mean age $=29.73, \mathrm{SD}=10.4)$ completed a modified German 17-item version of the TSK-G ${ }^{1}$ (Houben et al., 2005). The TSK-G consists of a 4-point Likert scale ranging from "strongly disagree" to "strongly agree" and includes questions such as "If I had LBP and I were to try to overcome it, my pain would increase." The questionnaire was originally validated in a Dutch sample of 2240 individuals divided in two groups of people with and without back complaints. Psychometric research indicated a sufficient reliability (Cronbach's $\alpha=0.78$ ) and high Tampa Scale for Kinesiophobia-G scores predicted pain catastrophising, pain intensity, pain-related health indices. Thus, the authors recommended the use of the TSK-G as a measure of FABs in general population studies (Houben et al., 2005). Furthermore, to control for possible influence of general anxiety, all subjects completed the State and Trait Anxiety Inventory (STAI) which is a common questionnaire that measures state and trait anxiety levels (Spielberger, 1971). Exclusion criteria were acute and/or recurrent back pain within the last 6 months, past chronic pain episodes, and a history of psychiatric or neurological disorders. Two subjects were excluded due to excessive head movements $(>2.5 \mathrm{~mm})$ during MR data acquisition, leaving a total sample size of 26 subjects for the final analysis. All subjects provided written informed consent for the participation in the experiment. The study was approved by the local ethics committee (Zurich, Switzerland) and was conducted in accordance with the Declaration of Helsinki.

For the statistical analyses, subjects were divided into groups with high and low TSK-G scores. TSK-G subgroups were defined using a median split (median $=35$ ), which resulted in 13 TSK$\mathrm{G}_{\text {low }}$ and 13 TSK-G $\mathrm{G}_{\text {high }}$ scorers. Groups were age- and gendermatched (Mann-Whitney $U$-test for age: $p=0.10$; chi-square test for gender: $p=0.31$ ).

\section{Scanning Parameters}

All measurements were performed on a 3-T whole-body MRI system (Philips Achieva, Best, the Netherlands), equipped with a 32-element receiving head coil and MultiTransmit parallel RF transmission. Each imaging session consisted of a survey scan, a B1 calibration scan (for MultiTransmit), a SENSE reference scan and a high resolution T1-weighted anatomical scan. fMRI data were acquired with whole-brain gradient-echo EPI sequences (365 volumes), consisting of 37 slices in the axial direction with the following parameters: field of view $=240 \mathrm{~mm} \times 240 \mathrm{~mm}$; acquisition matrix $=96 \times 96$; slice thickness $=2.8 \mathrm{~mm}$; interleaved slice acquisition; no slice gap; $\mathrm{TR}=2100 \mathrm{~ms}$; $\mathrm{TE}=30 \mathrm{~ms}$; SENSE factor $=2.5$; flip angel $80^{\circ}$. Anatomical data were obtained with a 3D T1-weighted turbo field echo scan consisting of 145 slices in sagittal orientation with the following parameters: field of view $=230 \mathrm{~mm} \times 226 \mathrm{~mm}$; slice thickness $=1.2 \mathrm{~mm}$; acquisition matrix $=208 \times 203$; repetition

${ }^{1}$ http://onlinelibrary.wiley.com/doi/10.1016/S1090-3801(09)60838-7/pdf 
time $=6.8 \mathrm{~ms}$; echo time $=3.1 \mathrm{~ms}$; flip angle $=9^{\circ}$; number of signal averages $=1$.

\section{Experimental Protocol}

The stimuli consisted of video clips with a duration of $4 \mathrm{~s}$ that showed potentially harmful activities for the back (shoveling soil with a bent back, lifting a flowerpot with slightly bent back and vacuum cleaning under a coffee table with a bent back) and harmless activities (walking up and down the stairs and walking on even ground). The videos were recorded from a third person perspective (Figure 1) and standardized in terms of duration of the potentially aversive movements. These daily activities were selected from the short electronic version of the Photograph Series of Daily Activities that has established a fear hierarchy of daily activities based on ratings of perceived harmfulness (Leeuw et al., 2007a). The video clips were displayed using MR-compatible goggles (Resonance Technology, Northridge, USA) connected to a computer running Presentation ${ }^{\circledR}$ software (Neurobehavioral Systems, Davis, CA, USA). Subjects were asked to carefully observe the video clips, which were shown in pseudo-randomized order (no more than two identical consecutive trials). The fMRI measurement with a total duration of $\sim 8$ min consisted of one fMRI session including 30 trials, and the three harmful and harmless activities were each presented five times. Immediately after the observation of the video clips, participants were asked to rate the perceived harmfulness of the activity on a visual analog scale (VAS). The VAS was anchored with the endpoints "not harmful at all" (0) and "extremely harmful" (10) and was shown for $4 \mathrm{~s}$. All ratings were performed using a MR compatible track ball (Current Desings, Philadelphia, PA, USA) that moved the indicator on the VAS scale. The duration of the

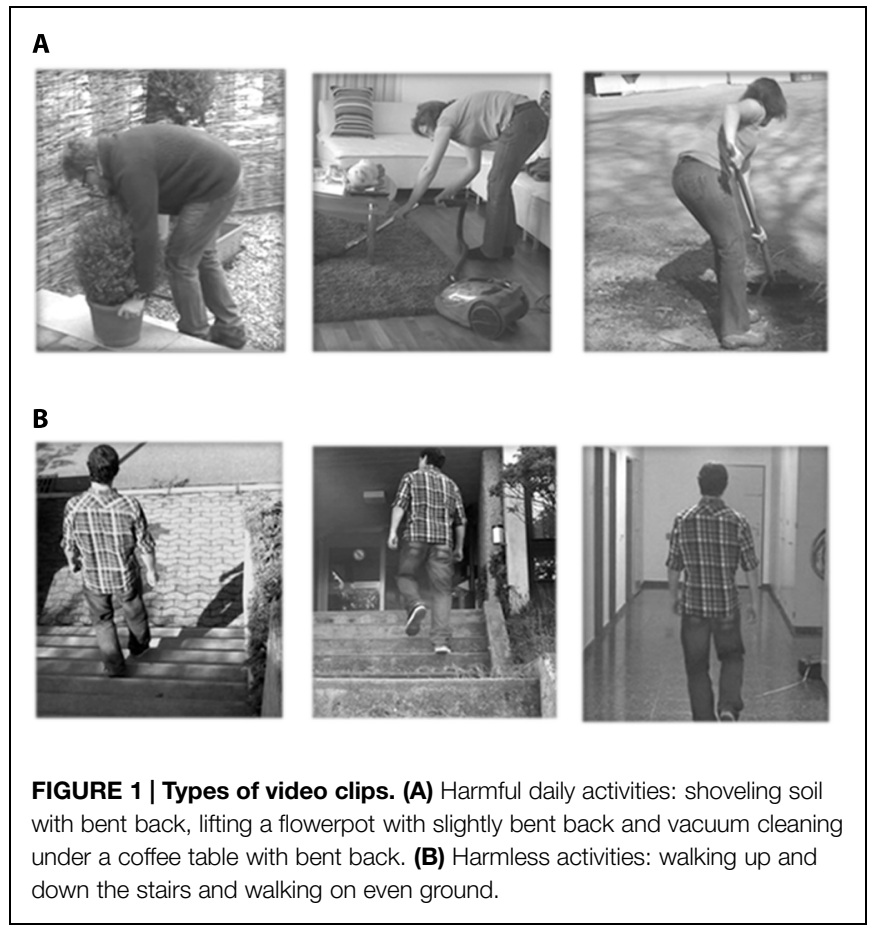

inter-stimulus interval (ISI, after the VAS rating, black screen with a green fixation cross) was jittered between 6 and $8 \mathrm{~s}$ and. The ISI was considered as a baseline, although there is no inherent baseline associated with the blood oxygen-leveldependent (BOLD) signal (Stark and Squire, 2001). We assumed that the baseline represented something akin to a zero-activity condition which was compared with activity during the different tasks.

\section{Image Preprocessing and Event-Related Analysis}

Except the preceding five dummy scans, all functional scans were included in the final analysis. Functional EPI volumes of each subject were corrected for differences in head motion, spatially normalized according to the Montreal Neurological Institute (MNI) space and finally smoothed with a $8 \mathrm{~mm}$ fullwidth at half-maximum (FWHM) Gaussian kernel. To control for confounding head movement effects, individual movement parameters (translations in $x, y$ and $z$-direction, as well as rotations around $x, y$, and $z$ axis) were implemented in the first level model as regressors of no interest. Excessive head motion was defined as a dislocation of more than once the in-plane voxel resolution $(>2.5 \mathrm{~mm})$. For removing the low frequency noise, a high-pass filter with a cut-off of $128 \mathrm{~s}$ was used. Trials were modeled as boxcar regressors and convolved with the standard canonical hemodynamic response function (HRF) as implemented in SPM8. For the first level analysis, the general linear model (GLM) was fitted for each subject by a design matrix composed of the onsets and duration (4s) of the pooled harmful and harmless video clips. For each subject, parameter estimates (beta) and contrast images (cons) were computed.

The resulting images were analyzed using a random-effects model to allow for population inferences (Friston et al., 1999). For the between group analyses, two-sample $t$-test were used, as implemented in SPM8. The variance between groups was assumed to be unequal. Error covariance components were estimated using restricted maximum likelihood, as implemented in SPM8. Activations and deactivations associated with the video clips were tested by simple positive and negative $t$-contrasts. Study independent amygdala masks were taken from the probabilistic Harvard-Oxford Cortical and Subcortical Structural Atlas $^{2}$. The probability threshold for belonging to the respective brain region was set to $p>0.5$. To control for false positives within the whole-brain results, we used cluster-based family-wise error correction (FWE) based on the Gaussian Random Field Theory (Chumbley and Friston, 2009). The identified clusters were considered to be significant if they fell below a clustercorrected $p(\mathrm{FWE})>0.05$. The resulting corrected SPM maps were extracted with $\mathrm{MarsBaR}^{3}$, color-coded and superimposed onto the MNI single-subject-T1 brain using MRIcroGL ${ }^{4}$. For percent signal change computations, $\operatorname{rfxplot}^{5}$ was used, and

\footnotetext{
${ }^{2} \mathrm{http}: / /$ www.fmrib.ox.ac.uk/fsl

${ }^{3}$ http://marsbar.sourceforge.net/

${ }^{4}$ http://www.cabiatl.com/mricrogl/

${ }^{5} \mathrm{http}: / /$ rfxplot.sourceforge.net/
} 
correlations were analyzed using Spearman's rank correlation coefficient (Glascher, 2009).

\section{Functional Connectivity}

The main advantage of psycho-physiological-interactions (PPIs) analysis is that it assesses co-variance between regions across time, and therefore provides a test of task effects on connectivity. For each subject, we extracted the deconvolved time course averaged across the bilateral amygdala clusters (identified by means of the amygdala probability masks, see above) of the contrast "harmful activities $>$ baseline," pooled across groups (the statistically more rigorous contrast "harmful activities $>$ harmless activities did not reveal any significant results). Subsequently, separate psychological terms (harmful and harmless video clips), physiological regressors (time course of seed region) and PPI interaction terms, as well as the movement parameters, were included in a generalized PPI model. The generalized form of the context-dependent PPI approach increases the flexibility of the statistical modeling and improves single-subject model-fit, thereby increasing the sensitivity to true positive findings and a reduction in false positives (McLaren et al., 2012). The resulting PPI connectivity estimates were then taken into a factorial design, as implemented in SPM8. Whole-brain functional connectivity analysis was performed using the bilateral amygdala cluster as a seed. Identified clusters were considered to be significant when falling below a cluster-corrected $p(\mathrm{FWE})<0.05$ (cluster extend was 65 voxels). An independent region of interest (ROI) for the pregenual anterior cingulate cortex (pgACC) was created using the MNI peak (4 40 12) with a $6 \mathrm{~mm}$ sphere reported in Loggia et al. (2013). Correlations between brain activity and TSK-G scores were analyzed using Spearman's rank correlation coefficient.

TABLE 1 | Cluster maxima of the event-related analysis (all clusters are listed here, minimum cluster size 10 voxels, height threshold $=p<0.001$, uncorrected), clusters which survived multiple comparisons family-wise error correction (FWE) are depicted in bold, MNI, Montreal Neurological Institute.

\begin{tabular}{|c|c|c|c|c|c|c|}
\hline \multirow[t]{2}{*}{ Cluster size } & \multirow[t]{2}{*}{$p$ (FWE) } & \multirow[t]{2}{*}{$\mathbf{T}$} & \multicolumn{3}{|c|}{ MNI coordinates } & \multirow[t]{2}{*}{ Brain region (AAL label) } \\
\hline & & & $x$ & $y$ & $\mathbf{z}$ & \\
\hline \multicolumn{7}{|c|}{ (A) TSK $_{\text {high }}$ "harmful activities < harmless activities" } \\
\hline 6258 & $<0.01$ & 7.76 & -26 & -52 & 72 & Left superior parietal lobe (Parietal_Sup_L) \\
\hline 750 & $<0.01$ & 7.94 & 54 & 26 & 18 & Right frontal lobe (Frontal_Inf_Tri_R) \\
\hline 1696 & $<0.01$ & 7.37 & 58 & -54 & 0 & Right middle temporal gyrus (Temporal_Mid_R) \\
\hline 595 & $<0.01$ & 6.46 & -20 & 0 & 68 & Left superior frontal gyrus (Frontal_Sup_L) \\
\hline 417 & $<0.01$ & 6.24 & -26 & 20 & -24 & Left inferior frontal gyrus (Frontal_Inf_Orb_L) \\
\hline 885 & $<0.01$ & 6.00 & 24 & -54 & 62 & Right superior parietal lobe (Parietal_Sup_R) \\
\hline 144 & $<0.01$ & 5.32 & 20 & 0 & -22 & Right parahippocampal gyrus (ParaHippocampal_R) \\
\hline 124 & $<0.01$ & 5.66 & -22 & -2 & -24 & Left amygdala (Amygdala_L) \\
\hline 51 & $<0.08$ & 5.16 & 30 & -30 & -8 & Right hippocampus (Hippocampus_R) \\
\hline 148 & $<0.01$ & 5.13 & -56 & 14 & 24 & Left inferior frontal gyrus (Frontal_Inf_Oper_L) \\
\hline 83 & $<0.05$ & 4.57 & 16 & -26 & 38 & Right precentral gyrus \\
\hline 47 & $<0.2$ & 4.30 & 66 & -18 & 32 & Right supramarginal gyrus (SupraMarginal_R) \\
\hline 23 & $<0.8$ & 4.29 & 2 & -2 & 38 & Right cingulate gyrus (Cingulum_Mid_R) \\
\hline 37 & $<0.1$ & 4.76 & 2 & 62 & 16 & Right medial frontal gyrus (Frontal_Sup_Medial_R) \\
\hline 52 & $<0.8$ & 4.35 & 62 & -36 & 8 & Right superior temporal gyrus (Temporal_Sup_R) \\
\hline 55 & $<0.2$ & 4.52 & -6 & 56 & 34 & Left medial frontal gyrus (Frontal_Sup_Medial_L) \\
\hline 23 & $<0.5$ & 3.96 & 24 & -76 & 46 & Right superior parietal lobe (Occipital_Sup_R) \\
\hline \multicolumn{7}{|c|}{ (B) TSK low "harmful activities > harmless activities" } \\
\hline 2016 & $<0.01$ & 8.42 & -48 & -26 & 34 & Left post-central gyrus (SupraMarginal_L) \\
\hline 683 & $<0.01$ & 6.35 & 24 & -54 & 62 & Right superior parietal lobe (Parietal_Sup_R) \\
\hline 3663 & $<0.01$ & 5.94 & -50 & -74 & -2 & Left inferior temporal gyrus (Occipital_Mid_L) \\
\hline 21 & $<0.7$ & 5.55 & -28 & -28 & -8 & Left hippocampus (Hippocampus_L) \\
\hline 85 & $<0.02$ & 5.23 & 22 & -84 & 46 & Right superior occipital gyrus (Cuneus_R) \\
\hline 57 & $<0.5$ & 4.60 & 54 & 22 & 10 & Right inferior frontal gyrus (Frontal_Inf_Tri_R) \\
\hline 77 & $<0.1$ & 4.41 & 64 & -36 & 20 & Right supramarginal gyrus (Temporal_Sup_R) \\
\hline 37 & $<0.5$ & 4.18 & 50 & -26 & 36 & Right post-central gyrus (SupraMarginal_R) \\
\hline 28 & $<0.8$ & 4.12 & 16 & -96 & 4 & Right cuneus (Calcarine_R) \\
\hline \multicolumn{7}{|c|}{ (C) TSK $_{\text {low }}>$ TSK $_{\text {high "harmful activities }}>$ harmless activities" } \\
\hline \multicolumn{7}{|c|}{ No significant clusters } \\
\hline \multicolumn{7}{|c|}{ 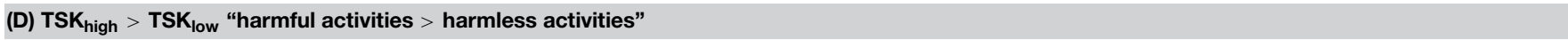 } \\
\hline 13 & $<0.1$ & 4.35 & -26 & 2 & -20 & Left amygdala (Amygdala_L) \\
\hline 16 & $<0.1$ & 4.03 & 20 & 4 & -24 & Right amygdala (Amygdala_R) \\
\hline
\end{tabular}




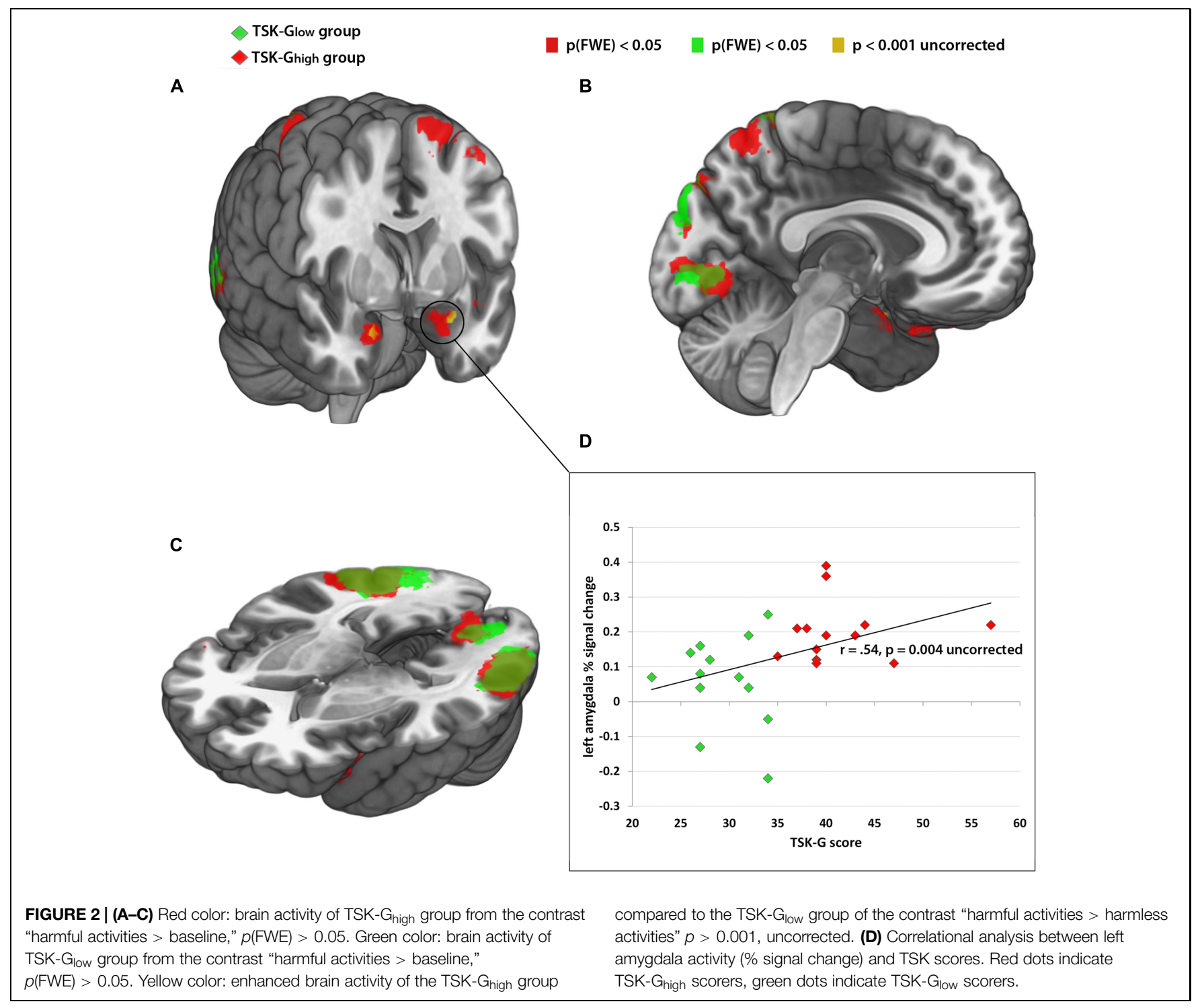

\section{Results}

\section{Behavioral Results}

The overall mean TSK-G score $(N=26)$ was 35.38 (SD $=1.52)$. TSK-G scores ranged from 22 to 57 points. The mean score of the TSK- $G_{\text {high }}$ group $(N=13)$ was $41.38(\mathrm{SD}=1.56)$ and for TSK$\mathrm{G}_{\text {low }}(N=13) 29.38(\mathrm{SD}=1.01)$. The VAS ratings were analyzed using a repeated-measures ANOVA with within-subject factor "video type" and between-subject factor "group." A significant effect of "video type" could be observed $[F(1,24)=10.74$, $p=0.04]$ whereas neither an effect of "group" $[F(1,24)=2.54$, $p=0.12]$ nor an interaction effect "group $\times$ video type" could be detected $[F(1,24)=0.31, p=0.58]$. The post hoc $t$-test revealed that the potentially harmful activities were rated as more hazardous than the harmless activities $[t(25)=9.98$, $p=0.01$, mean rating harmful $=4.81, \mathrm{SD}=1.8$, mean rating harmless $=1.2, \mathrm{SD}=1.2]$. The overall mean state score was 43.19 $(\mathrm{SD}=4.18)$ whereas the mean trait score was $42.80(\mathrm{SD}=3.24)$.
Importantly, state and trait anxiety levels did not differ between the high and low TSK-G groups [Mean scores: TSK- $\mathrm{G}_{\text {high }}$ group: state $(43.38, \mathrm{SD}=4.73)$ trait $(43.46, \mathrm{SD}=3.38) / \mathrm{TSK}-\mathrm{G}_{\mathrm{low}}$ group: state $(43.00, \mathrm{SD}=3.74)$ trait $(42.15, \mathrm{SD}=3.10) /$ Two sample $t$-test, state: $t(24)=0.23, p=0.82$; trait: $t(24)=1.03, p=0.32$ ]. Moreover, state scores did not correlate with TSK-G scores (Pearson's $r=-0.47, p=0.82$ ) whereas trait scores showed a statistical trend (Pearson's $r=0.34, p=0.09$, uncorrected). These findings indicate a distinct and state-trait anxiety independent role of movement-related fear of pain as assessed by the TSK-G questionnaire.

\section{Functional Imaging Results}

The categorical whole-brain analysis of the contrast "harmful activities > harmless activities" yielded significant bilateral amygdala activity in the TSK- $G_{\text {high }}$ group, whereas no amygdala activity was observed in the TSK-G $\mathrm{G}_{\text {low }}$ group (peak MNI coordinates: left amygdala $-22-2-24$, right amygdala: 200 
TABLE 2 | Cluster maxima of the functional connectivity analysis with the bilateral amygdala as a seed (all clusters are listed here, minimum cluster size 10 voxels, height threshold $=p<\mathbf{0 . 0 0 1}$, uncorrected, clusters which survived multiple comparisons FWE are depicted in bold), MNI, Montreal Neurological Institute.

\begin{tabular}{|c|c|c|c|c|c|c|}
\hline \multirow[t]{2}{*}{ Cluster size } & \multirow[t]{2}{*}{$p$ (FWE) } & \multirow[t]{2}{*}{$\mathbf{T}$} & \multicolumn{3}{|c|}{ MNI coordinates (mm) } & \multirow[t]{2}{*}{ Brain region (AAL label) } \\
\hline & & & $\mathbf{x}$ & $\mathbf{y}$ & $\mathbf{z}$ & \\
\hline \multicolumn{7}{|c|}{ (A) TSK $_{\text {high }}$ "harmful activites > baseline" } \\
\hline 217 & $<0.01$ & 7.98 & 18 & -8 & -22 & Right parahippocampal gyrus (ParaHippocampal_R) \\
\hline 291 & $<0.01$ & 5.98 & 38 & -64 & 20 & Right middle temporal gyrus \\
\hline 131 & $<0.01$ & 5.70 & 38 & -46 & -14 & Right fusiform gyrus (Fusiform_R) \\
\hline 12 & $<0.1$ & 5.52 & 20 & 20 & 28 & Right frontal lobe \\
\hline 137 & $<0.01$ & 5.36 & -20 & -6 & -22 & Left hippocampus (Hippocampus_L) \\
\hline 32 & $<0.4$ & 5.04 & -36 & -66 & -4 & Left occipital lobe \\
\hline 50 & $<0.1$ & 4.47 & -46 & -62 & 16 & Left middle occipital gyrus (Temporal_Mid_L) \\
\hline 16 & $<0.1$ & 4.38 & -18 & -46 & 0 & Left precuneus (Precuneus_L) \\
\hline 10 & $<0.1$ & 4.33 & -48 & -80 & 14 & Left inferior occipital gyrus (Occipital_Mid_L) \\
\hline 41 & $<0.2$ & 4.22 & -62 & -28 & 44 & Left post-central gyrus \\
\hline 12 & $<0.1$ & 4.22 & -30 & -18 & -20 & Left hippocampus (Hippocampus_L) \\
\hline 18 & $<0.9$ & 4.03 & 56 & -48 & 6 & Right middle temporal gyrus (Temporal_Mid_R) \\
\hline 14 & $<0.1$ & 3.99 & -6 & -32 & -6 & Left brain stem \\
\hline 12 & $<0.1$ & 3.94 & -4 & -56 & -4 & Left cerebellum (Cerebelum_4_5_L) \\
\hline \multicolumn{7}{|c|}{ (B) TSK $_{\text {low }}$ "harmful activites > baseline" } \\
\hline 160 & $<0.01$ & 6.64 & 18 & -6 & -20 & Right parahippocampal gyrus (ParaHippocampal_R) \\
\hline 597 & $<0.01$ & 6.42 & -6 & 36 & 18 & Left anterior cingulate (Cingulum_Ant_L) \\
\hline 165 & $<0.01$ & 5.16 & 6 & 36 & 2 & Right anterior cingulate (Cingulum_Ant_R) \\
\hline 32 & $<0.4$ & 5.08 & -12 & 18 & -4 & Left caudate (Caudate_L) \\
\hline 180 & $<0.01$ & 5.01 & 14 & -56 & 22 & Right precuneus (Precuneus_R) \\
\hline 79 & $<0.01$ & 4.95 & -44 & 12 & -16 & Left superior temporal gyrus (Temporal_Pole_Sup_L) \\
\hline 40 & $<0.20$ & 4.92 & 52 & 38 & 0 & Right inferior frontal gyrus (Frontal_Inf_Tri_R) \\
\hline 54 & $<0.08$ & 4.78 & -10 & 8 & -14 & Left nucleus accumbens (Olfactory_L) \\
\hline 58 & $<0.05$ & 4.68 & 56 & 24 & 2 & Right inferior frontal gyrus (Frontal_Inf_Tri_R) \\
\hline 31 & $<0.5$ & 4.40 & -42 & 16 & 2 & Left insula (Insula_L) \\
\hline 27 & $<0.6$ & 4.37 & -54 & 8 & 38 & Left middle frontal gyrus (Precentral_L) \\
\hline 26 & $<0.6$ & 4.33 & -44 & -82 & 20 & Left middle occipital gyrus (Occipital_Mid_L) \\
\hline 35 & $<0.3$ & 4.31 & 54 & -66 & 22 & Right middle temporal gyrus (Temporal_Mid_R) \\
\hline 15 & $<0.1$ & 4.26 & 56 & 14 & 22 & Right inferior frontal gyrus (Frontal_Inf_Oper_R) \\
\hline 12 & $<0.1$ & 4.24 & 6 & 8 & 72 & Right superior frontal gyrus (Supp_Motor_Area_R) \\
\hline 20 & $<0.8$ & 4.15 & -56 & 10 & 24 & Left inferior frontal gyrus (Frontal_Inf_Oper_L) \\
\hline 10 & $<0.9$ & 4.10 & 10 & 6 & -16 & Right lateral front-orbital gyrus \\
\hline 11 & $<0.9$ & 4.09 & 46 & 20 & -12 & Right superior temporal gyrus (Frontal_Inf_Orb_R) \\
\hline 13 & $<0.9$ & 4.04 & 0 & 12 & 46 & Left medial frontal gyrus (Supp_Motor_Area_L) \\
\hline 10 & $<0.9$ & 3.93 & -52 & -26 & 28 & Left inferior parietal lobe (SupraMarginal_L) \\
\hline \multicolumn{7}{|c|}{ 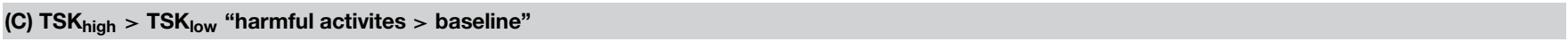 } \\
\hline \multicolumn{7}{|c|}{ No significant clusters } \\
\hline \multicolumn{7}{|c|}{ 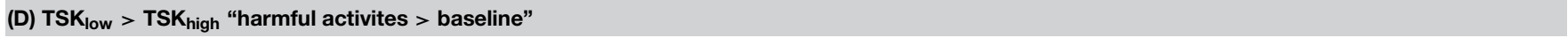 } \\
\hline 65 & $<0.03$ & 6.02 & 12 & 38 & 0 & Right anterior cingulate (Cingulum_Ant_R) \\
\hline 51 & $<0.1$ & 5.32 & -6 & 36 & 18 & Left anterior cingulate (Cingulum_Ant_L) \\
\hline 24 & $<0.7$ & 4.63 & -28 & 54 & 16 & Left middle frontal gyrus (Front_Mid_L) \\
\hline 20 & $<0.8$ & 4.43 & 44 & 26 & 0 & Left inferior frontal gyrus (Frontal_Inf_Tri_R) \\
\hline 11 & $<0.9$ & 4.23 & -12 & 18 & -2 & Left caudate (Caudate_L) \\
\hline 14 & $<0.9$ & 4.17 & 10 & 24 & 44 & Right medial frontal gyrus (Frontal_Sup_Medial_R) \\
\hline 24 & $<0.7$ & 4.02 & -42 & 8 & -12 & Left insula (Insula_L) \\
\hline
\end{tabular}

$-22, p(\mathrm{FWE})<0.05$, Tables 1A,B; Figures 2A-C). A direct whole-brain comparison (two-sample $t$-test) of the contrast "harmful activities harmless activities" between TSK-G $G_{\text {high }}$ and TSK- $\mathrm{G}_{\text {low }}$ groups revealed bilateral activity exclusively in the amygdala ( $p<0.001$, uncorrected, Figure 2A; Tables 1C,D). Furthermore, left amygdala responses (\% signal change) to the harmful activities relative to baseline positively correlated with the TSK-G score ( $r=0.54, p=0.004$, uncorrected, Figure 2D). 

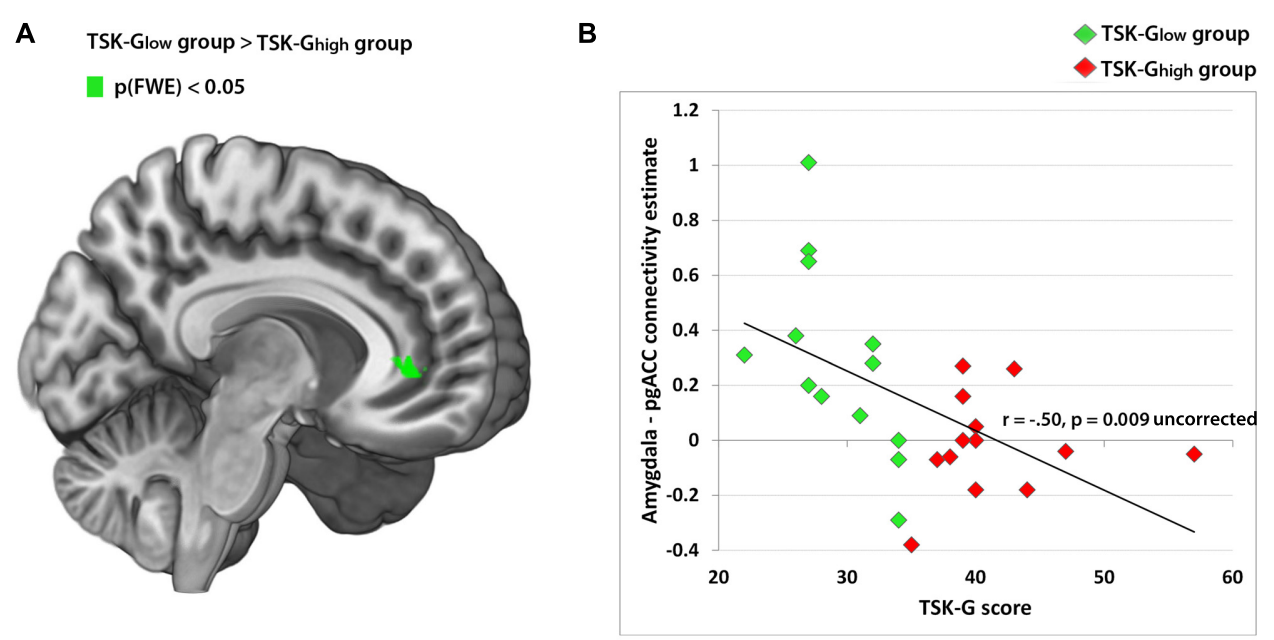

FIGURE 3 | (A) Amygdala-pgACC functional connectivity cluster (TSK-Glow > TSK-G high from the contrast "harmful activities > baseline"), peak MNI 12 38 0, $p$ (FWE) < 0.05. (B) Correlation analysis of amygdala-pgACC (Loggia et al., 2013; pgACC peak) functional connectivity and TSK scores. Red dots indicate TSK-Ghigh scorers, green dots indicate TSK-Glow scorers.

No significant correlations were detected for the right amygdala or for harmless activities (all $p$-values $>0.19$ ). Importantly, no significant relationships were detected between left and right amygdala activity and individual state (both $p$-values $>0.1$ ) and trait scores (both $p$-values $>0.7$ ).

\section{Functional Connectivity Results}

Using the amygdala as a seed, the whole-brain analysis of the contrast "harmful activities $>$ baseline" revealed no significant enhanced connectivity of the TSK-G $\mathrm{G}_{\text {high }}$ group compared to the TSK-G $\mathrm{G}_{\text {low }}$ group. However, the reverse comparison (TSK$\mathrm{G}_{\text {low }}>$ TSK- $\mathrm{G}_{\text {high }}$ ) yielded a significant cluster in the pgACC [Peak MNI 1238 0, p(FWE) $<0.05$, Table 2; Figure 3A]. The respective correlation analysis using the study-independent pgACC ROI (Loggia et al., 2013) revealed a significant and negative relationship between the amygdala/pgACC functional coupling strength and the TSK-G score $(r=-0.50, p=0.009$, uncorrected, Figure 3B). Further correlational analyses of connectivity estimates between these two regions did not show significant correlations between the contrast "harmless activities > baseline" and TSK-G scores or the state and trait scores (all $p$-values $>0.11$ ).

\section{Discussion}

Personality and psychosocial environment factors seem to play an important role in the variability in individual resilience to dysfunctional alterations in the presence of pain. Specific psychological processes and underlying brain networks may be involved in conferring vulnerability to painful conditions that likely contribute to some of these discrepancies (Denk et al., 2014; Simons et al., 2014a).The most consistent finding is the strong predictive power of FABs and pain-related fear for perceived disability in chronic pain (Vlaeyen et al., 1995a,b;
Cook et al., 2006). Although not everyone agrees that pain-related fear should be considered as a phobia, pain researchers and clinicians alike do agree on the importance of pain-related fear and FABs in explaining disabilities and the transition from acute to chronic musculoskeletal pain (Vlaeyen et al., 1995a,b; Vlaeyen and Linton, 2000; Houben et al., 2005; Wertli et al., 2013). However, no neural substrates of back pain related FABs have been found to date (Barke et al., 2012). The results of the current study demonstrate that the observation of potentially harmful daily activities induced enhanced amygdala activity in pain-free individuals without a history of chronic LBP episodes but with elevated fear of movement, as measured by the TSK$\mathrm{G}$ questionnaire. The functional connectivity analysis yielded a reduced functional amygdala - pgACC coupling in TSK-G $\mathrm{G}_{\text {high }}$ scorers.

Event-related and connectivity measures showed significant, although divergent, correlations between the brain activity induced by the observation of potentially harmful daily activities and TSK-G scores. Enhanced amygdala activity was positively correlated with TSK-G scores, whereas stronger amygdalapgACC coupling was negatively associated with TSK-G scores. Importantly, neither state anxiety nor trait anxiety scores showed a significant relationship with brain activity or functional connectivity. These findings demonstrate the stimulus-specific role of task-related amygdala reactivity and movement-related fear of pain.

Increased affective responses involve bottom-up emotional processes that are mediated by subcortical structures, amongst which the amygdala plays a key role (Seifritz et al., 2003; Wager et al., 2004; Ellingsen et al., 2013; Denk et al., 2014; Silvers et al., 2014). The amygdala activation observed in the current study is consistent with models that implicate this brain region in the evaluation and representation of perceived fear intensity and pain, as well as contributing to the deciphering of threats in visual scenes (Goossens et al., 2007; Brugger et al., 2011; 
Kryklywy et al., 2013). Furthermore, accumulating evidence points to the amygdala as an important site for a reciprocal relationship between persistent pain and negative affective states such as fear and anxiety (Neugebauer et al., 2004). Likewise, the elicitation of negative affect and pain consistently activates the pgACC (Shackman et al., 2011). The amygdala maintains intensive crosstalk with forebrain regions, such as the anterior cingulate cortex (ACC) and prefrontal regions (e.g., pgACC, vmPFC, and the dorsolateral prefrontal cortex, DLPFC); this crosstalk forms a neural circuit involved in emotional control and modulation of pain within the descending pain modulatory system (DPMS; Bushnell et al., 2013; Denk et al., 2014). The DPMS constitutes a powerful neural circuit that regulates nociceptive processing in the dorsal horn of the spinal cord and thereby controls which signals enter the brain. As such, it plays an important role in modulating the eventual pain experience (Tracey and Dickenson, 2012; Denk et al., 2014).

Furthermore, there is unique evidence for the critical role of the pgACC in pain inhibition forming an anti-nociceptive network involving subcortical structures such as the amygdala and the periaqueductal gray (PAG; Petrovic et al., 2002; Wager et al., 2004; Bingel et al., 2006; Eippert et al., 2009; Loggia et al., 2013). Previous studies demonstrated that placebo analgesia increases functional coupling between the pgACC and subcortical structures such as the amygdala (Ellingsen et al., 2013). Furthermore, pgACC connectivity with the default-mode network (DMN) has been associated with pain-protective effects (Loggia et al., 2013). Hence, the observed stimulus specific hyperreactivity of the amygdala, and its reduced functional coupling to the pgACC, in individuals with elevated FABs might indicate a premorbid neural mechanism in a pain-free state. This finding may confer a vulnerability of TSK-G $\mathrm{G}_{\text {high }}$ scorers to a possible transition from acute to chronic state in future LBP episodes.

Although differences on a neural level were demonstrated, both groups rated the potential harmfulness of activities equivalently. In addition to a possible lack of sensitivity of the applied VAS scale, the absence of group differences on a behavioral level may indicate an unconscious neural process. This theory is in line with investigations that have demonstrated that the amygdala and medial prefrontal brain regions can be activated and exhibit automatic responses that are outside the focus of attention and conscious processes (Vuilleumier et al., 2002; Bryant et al., 2008). Furthermore, it has been proposed that this network provides a rapid and automatic alerting mechanism

\section{References}

Baliki, M. N., Petre, B., Torbey, S., Herrmann, K. M., Huang, L., Schnitzer, T. J., et al. (2012). Corticostriatal functional connectivity predicts transition to chronic back pain. Nat. Neurosci. 15, 1117-1119. doi: 10.1038/nn.3153

Barke, A., Baudewig, J., Schmidt-Samoa, C., Dechent, P., and Kroner-Herwig, B. (2012). Neural correlates of fear of movement in high and low fear-avoidant chronic low back pain patients: an event-related fMRI study. Pain 153, 540-552. doi: 10.1016/j.pain.2011.11.012

Bingel, U., Lorenz, J., Schoell, E., Weiller, C., and Buchel, C. (2006). Mechanisms of placebo analgesia: rACC recruitment of a subcortical antinociceptive network. Pain 120, 8-15. doi: 10.1016/j.pain.2005.08.027 for responding to unconscious signals of fear that is "vital for the automatic orienting of attention toward the stimulus and to highlight the stimulus for further cognitive evaluation" (Liddell et al., 2005; Bryant et al., 2008).

However, whether the origin of these alterations in TSK- $\mathrm{G}_{\text {high }}$ scorers are either inherent or maladaptive remains elusive (Denk et al., 2014). In the general population, large surveys have shown that a range of erroneous back pain-related FABs exist that have arisen from social means, including fear inducing information, and vicarious exposure, such as observations, regardless of the presence of back pain (Buchbinder et al., 2001, 2008; Gross et al., 2006). On the neural level, Phelps et al. (2001) have demonstrated that fears acquired through verbal instruction were associated with robust activation in the amygdala. Interestingly, this activation was predominantly observed in the left amygdala, and thus corresponds to the results of the current study, where the TSK-G scores were only significantly correlated with activity in the left amygdala. These results indicate a left amygdalar dominance of back pain-related fears, which might be acquired through social and/or instructed fear learning. Further support for a left amygdalar dominance in the physiological expression of instructed fear learning comes from subjects with unilateral amygdala damage (Olsson and Phelps, 2007). However, further studies on instructed fear are needed to confirm this left dominance of the amygdala.

A growing body of evidence points to psychological factors, in particular FABs that serve as strong predictors of disability and chronification in LBP. The current investigation provides initial evidence for a correlative neurobiological substrate that might reflect premorbid brain network properties underlying psychological factors that may become relevant for enhanced disability in a future LBP episode. This finding may lead to a more mechanistic understanding on the neural level that supports preventative psychology-based interventions in acute episodes or in exposure in vivo in individuals with elevated FABs. Furthermore, there seem to be a call for greater public education in terms of erroneous beliefs regarding back pain. However, the current findings have to be carefully interpreted. A detailed understanding whether changes in these brain signatures are causal in producing cLBP is currently far impossible as it relies on the detailed knowledge of biological and environmental subject variables (Denk et al., 2014). Further studies based on longterm observations including patients are necessary to establish a causal relationship between the indicated brain signatures as a vulnerability factor for chronic LBP.

Bronfort, G., Haas, M., Evans, R. L., and Bouter, L. M. (2004). Efficacy of spinal manipulation and mobilization for low back pain and neck pain: a systematic review and best evidence synthesis. Spine J. 4, 335-356. doi: 10.1016/j.spinee.2003.06.002

Brugger, M., Ettlin, D. A., Meier, M., Keller, T., Luechinger, R., Barlow, A., et al. (2011). Taking sides with pain - Lateralization aspects related to cerebral processing of dental pain. Front. Hum. Neurosci. 5:12. doi: 10.3389/fnhum.2011.00012

Bryant, R. A., Kemp, A. H., Felmingham, K. L., Liddell, B., Olivieri, G., Peduto, A., et al. (2008). Enhanced amygdala and medial prefrontal activation during nonconscious processing of fear in posttraumatic stress disorder: an fMRI study. Hum. Brain Mapp. 29, 517-523. doi: 10.1002/hbm.20415 
Buchbinder, R., Gross, D. P., Werner, E. L., and Hayden, J. A. (2008). Understanding the characteristics of effective mass media campaigns for back pain and methodological challenges in evaluating their effects. Spine (Phila $\mathrm{Pa}$ 1976) 33, 74-80. doi: 10.1097/BRS.0b013e31815e39c8

Buchbinder, R., Jolley, D., and Wyatt, M. (2001). 2001 Volvo Award Winner in Clinical Studies: effects of a media campaign on back pain beliefs and its potential influence on management of low back pain in general practice. Spine (Phila Pa 1976) 26, 2535-2542. doi: 10.1097/00007632-200112010-00005

Buer, N., and Linton, S. J. (2002). Fear-avoidance beliefs and catastrophizing: occurrence and risk factor in back pain and $\mathrm{ADL}$ in the general population. Pain 99, 485-491. doi: 10.1016/S0304-3959(02)00265-8

Bushnell, M. C., Ceko, M., and Low, L. A. (2013). Cognitive and emotional control of pain and its disruption in chronic pain. Nat. Rev. Neurosci. 14, 502-511. doi: $10.1038 / \mathrm{nrn} 3516$

Callan, D., Mills, L., Nott, C., England, R., and England, S. (2014). A tool for classifying individuals with chronic back pain: using multivariate pattern analysis with functional magnetic resonance imaging data. PLoS ONE 9:e98007. doi: 10.1371/journal.pone.0098007

Chou, R., and Shekelle, P. (2010). Will this patient develop persistent disabling low back pain? JAMA 303, 1295-1302. doi: 10.1001/jama.2010.344

Chumbley, J. R., and Friston, K. J. (2009). False discovery rate revisited: FDR and topological inference using Gaussian random fields. Neuroimage 44, 62-70. doi: 10.1016/j.neuroimage.2008.05.021

Cook, A. J., Brawer, P. A., and Vowles, K. E. (2006). The fear-avoidance model of chronic pain: validation and age analysis using structural equation modeling. Pain 121, 195-206. doi: 10.1016/j.pain.2005.11.018

Denk, F., Mcmahon, S. B., and Tracey, I. (2014). Pain vulnerability: a neurobiological perspective. Nat. Neurosci. 17, 192-200. doi: 10.1038/nn.3628

Dunn, K. M., Hestbaek, L., and Cassidy, J. D. (2013). Low back pain across the life course. Best Pract. Res. Clin. Rheumatol. 27, 591-600. doi: 10.1016/j.berh.2013.09.007

Eippert, F., Bingel, U., Schoell, E. D., Yacubian, J., Klinger, R., Lorenz, J., et al. (2009). Activation of the opioidergic descending pain control system underlies placebo analgesia. Neuron 63, 533-543. doi: 10.1016/j.neuron.2009.07.014

Ellingsen, D. M., Wessberg, J., Eikemo, M., Liljencrantz, J., Endestad, T., Olausson, H., et al. (2013). Placebo improves pleasure and pain through opposite modulation of sensory processing. Proc. Natl. Acad. Sci. U.S.A. 110, 17993-17998. doi: 10.1073/pnas.1305050110

Friston, K. J., Holmes, A. P., Price, C. J., Buchel, C., and Worsley, K. J. (1999). Multisubject fMRI studies and conjunction analyses. Neuroimage 10, 385-396. doi: 10.1006/nimg.1999.0484

Glascher, J. (2009). Visualization of group inference data in functional neuroimaging. Neuroinformatics 7, 73-82. doi: 10.1007/s12021-008-9042-x

Goossens, L., Sunaert, S., Peeters, R., Griez, E. J., and Schruers, K. R. (2007). Amygdala hyperfunction in phobic fear normalizes after exposure. Biol. Psychiatry 62, 1119-1125. doi: 10.1016/j.biopsych.2007.04.024

Gross, D. P., Ferrari, R., Russell, A. S., Battie, M. C., Schopflocher, D., Hu, R. W., et al. (2006). A population-based survey of back pain beliefs in Canada. Spine (Phila Pa 1976) 31, 2142-2145. doi: 10.1097/01.brs.0000231771.14965.e4

Hashmi, J. A., Baliki, M. N., Huang, L., Baria, A. T., Torbey, S., Hermann, K. M., et al. (2013). Shape shifting pain: chronification of back pain shifts brain representation from nociceptive to emotional circuits. Brain 136, 2751-2768. doi: 10.1093/brain/awt211

Houben, R. M., Leeuw, M., Vlaeyen, J. W., Goubert, L., and Picavet, H. S. (2005). Fear of movement/injury in the general population: factor structure and psychometric properties of an adapted version of the Tampa Scale for Kinesiophobia. J. Behav. Med. 28, 415-424. doi: 10.1007/s10865-0059011-x

Ivo, R., Nicklas, A., Dargel, J., Sobottke, R., Delank, K. S., Eysel, P., et al. (2013). Brain structural and psychometric alterations in chronic low back pain. Eur. Spine J. 22, 1958-1964. doi: 10.1007/s00586-013-2692-x

Koes, B. W., Van Tulder, M. W., Ostelo, R., Kim Burton, A., and Waddell, G. (2001). Clinical guidelines for the management of low back pain in primary care: an international comparison. Spine (Phila Pa 1976) 26, 2504-2513; discussion 2513-2504. doi: 10.1097/00007632-200111150-00022

Kryklywy, J. H., Nantes, S. G., and Mitchell, D. G. (2013). The amygdala encodes level of perceived fear but not emotional ambiguity in visual scenes. Behav. Brain Res. 252, 396-404. doi: 10.1016/j.bbr.2013.06.010
Leeuw, M., Goossens, M. E., Van Breukelen, G. J., Boersma, K., and Vlaeyen, J. W. (2007a). Measuring perceived harmfulness of physical activities in patients with chronic low back pain: the Photograph Series of Daily Activities-short electronic version. J. Pain 8, 840-849. doi: 10.1016/j.jpain.2007.05.013

Leeuw, M., Houben, R. M., Severeijns, R., Picavet, H. S., Schouten, E. G., and Vlaeyen, J. W. (2007b). Pain-related fear in low back pain: a prospective study in the general population. Eur. J. Pain 11, 256-266. doi: 10.1016/j.ejpain.2006.02.009

Liddell, B. J., Brown, K. J., Kemp, A. H., Barton, M. J., Das, P., Peduto, A., et al. (2005). A direct brainstem-amygdala-cortical 'alarm' system for subliminal signals of fear. Neuroimage 24, 235-243. doi: 10.1016/j.neuroimage.2004.08.016

Lloyd, D. M., Findlay, G., Roberts, N., and Nurmikko, T. (2014). Illness behavior in patients with chronic low back pain and activation of the affective circuitry of the brain. Psychosom. Med. 76, 413-421. doi: 10.1097/PSY.0000000000000076

Loggia, M. L., Kim, J., Gollub, R. L., Vangel, M. G., Kirsch, I., Kong, J., et al. (2013). Default mode network connectivity encodes clinical pain: an arterial spin labeling study. Pain 154, 24-33. doi: 10.1016/j.pain.2012.07.029

Mansour, A. R., Baliki, M. N., Huang, L., Torbey, S., Herrmann, K. M., Schnitzer, T. J., et al. (2013). Brain white matter structural properties predict transition to chronic pain. Pain 154, 2160-2168. doi: 10.1016/j.pain.2013.06.044

McLaren, D. G., Ries, M. L., Xu, G., and Johnson, S. C. (2012). A generalized form of context-dependent psychophysiological interactions (gPPI): a comparison to standard approaches. Neuroimage 61, 1277-1286. doi: 10.1016/j.neuroimage.2012.03.068

Neugebauer, V., Li, W., Bird, G. C., and Han, J. S. (2004). The amygdala and persistent pain. Neuroscientist 10, 221-234. doi: 10.1177/1073858403261077

Olsson, A., and Phelps, E. A. (2007). Social learning of fear. Nat. Neurosci. 10, 1095-1102. doi: $10.1038 / \mathrm{nn} 1968$

Peterson, C. K., Bolton, J., and Humphreys, B. K. (2012). Predictors of improvement in patients with acute and chronic low back pain undergoing chiropractic treatment. J. Manipulative Physiol. Ther. 35, 525-533. doi: 10.1016/j.jmpt.2012.06.003

Petrovic, P., Kalso, E., Petersson, K. M., and Ingvar, M. (2002). Placebo and opioid analgesia - imaging a shared neuronal network. Science 295, 1737-1740. doi: 10.1126/science. 1067176

Phelps, E. A., O'Connor, K. J., Gatenby, J. C., Gore, J. C., Grillon, C., and Davis, M. (2001). Activation of the left amygdala to a cognitive representation of fear. Nat. Neurosci. 4, 437-441. doi: 10.1038/86110

Rainville, J., Smeets, R. J., Bendix, T., Tveito, T. H., Poiraudeau, S., and Indahl, A. J. (2011). Fear-avoidance beliefs and pain avoidance in low back pain - translating research into clinical practice. Spine J. 11, 895-903. doi: 10.1016/j.spinee.2011.08.006

Rapoport, J., Jacobs, P., Bell, N. R., and Klarenbach, S. (2004). Refining the measurement of the economic burden of chronic diseases in Canada. Chronic Dis. Can. 25, 13-21.

Seifritz, E., Esposito, F., Neuhoff, J. G., Luthi, A., Mustovic, H., Dammann, G., et al. (2003). Differential sex-independent amygdala response to infant crying and laughing in parents versus nonparents. Biol. Psychiatry 54, 1367-1375. doi: 10.1016/S0006-3223(03)00697-8

Shackman, A. J., Salomons, T. V., Slagter, H. A., Fox, A. S., Winter, J. J., and Davidson, R. J. (2011). The integration of negative affect, pain and cognitive control in the cingulate cortex. Nat. Rev. Neurosci. 12, 154-167. doi: $10.1038 / \mathrm{nrn} 2994$

Silvers, J. A., Wager, T. D., Weber, J., and Ochsner, K. N. (2014). The neural bases of uninstructed negative emotion modulation. Soc. Cogn. Affect. Neurosci. 10, 10-18. doi: $10.1093 / \mathrm{scan} / \mathrm{nsu} 016$

Simons, L. E., Elman, I., and Borsook, D. (2014a). Psychological processing in chronic pain: a neural systems approach. Neurosci. Biobehav. Rev. 39, 61-78. doi: 10.1016/j.neubiorev.2013.12.006

Simons, L. E., Pielech, M., Erpelding, N., Linnman, C., Moulton, E., Sava, S., et al. (2014b). The responsive amygdala: treatment-induced alterations in functional connectivity in pediatric complex regional pain syndrome. Pain 155, 1727-1742. doi: 10.1016/j.pain.2014.05.023

Spielberger, C. D. (1971). Notes and comments trait-state anxiety and motor behavior. J. Mot. Behav. 3, 265-279. doi: 10.1080/00222895.1971.10734907

Stark, C. E., and Squire, L. R. (2001). When zero is not zero: the problem of ambiguous baseline conditions in fMRI. Proc. Natl. Acad. Sci. U.S.A. 98, 12760-12766. doi: 10.1073/pnas.221462998 
Tracey, I., and Dickenson, A. (2012). SnapShot: pain perception. Cell 148, 1308-1308.e2. doi: 10.1016/j.cell.2012.03.004

Ung, H., Brown, J. E., Johnson, K. A., Younger, J., Hush, J., and Mackey, S. (2014). Multivariate classification of structural MRI data detects chronic low back pain. Cereb. Cortex 24, 1037-1044. doi: 10.1093/cercor/bhs378

Vlaeyen, J. W., Kole-Snijders, A. M., Boeren, R. G., and Van Eek, H. (1995a). Fear of movement/(re)injury in chronic low back pain and its relation to behavioral performance. Pain 62, 363-372. doi: 10.1016/0304-3959(94)00279-N

Vlaeyen, J. W., Kole-Snijders, A. M., Rotteveel, A. M., Ruesink, R., and Heuts, P. H. (1995b). The role of fear of movement/(re)injury in pain disability. J. Occup. Rehabil. 5, 235-252. doi: 10.1007/BF02109988

Vlaeyen, J. W., and Linton, S. J. (2000). Fear-avoidance and its consequences in chronic musculoskeletal pain: a state of the art. Pain 85, 317-332. doi: 10.1016/S0304-3959(99)00242-0

Vuilleumier, P., Armony, J. L., Clarke, K., Husain, M., Driver, J., and Dolan, R. J. (2002). Neural response to emotional faces with and without awareness: event-related fMRI in a parietal patient with visual extinction and spatial neglect. Neuropsychologia 40, 2156-2166. doi: 10.1016/S0028-3932(02) 00045-3
Wager, T. D., Rilling, J. K., Smith, E. E., Sokolik, A., Casey, K. L., Davidson, R. J., et al. (2004). Placebo-induced changes in FMRI in the anticipation and experience of pain. Science 303, 1162-1167. doi: 10.1126/science.1093065

Wertli, M.M., Rasmussen-Barr, E., Weiser, S., Bachmann, L.M., and Brunner, F. (2013). The role of fear avoidance beliefs as a prognostic factor for outcome in patients with nonspecific low back pain: a systematic review. Spine J. 14, 816-836. doi: 10.1016/j.spinee.2013.09.036

Conflict of Interest Statement: The authors declare that the research was conducted in the absence of any commercial or financial relationships that could be construed as a potential conflict of interest.

Copyright (C) 2015 Meier, Stämpfli, Vrana, Humphreys, Seifritz and HotzBoendermaker. This is an open-access article distributed under the terms of the Creative Commons Attribution License (CC BY). The use, distribution or reproduction in other forums is permitted, provided the original author(s) or licensor are credited and that the original publication in this journal is cited, in accordance with accepted academic practice. No use, distribution or reproduction is permitted which does not comply with these terms. 